\title{
COMPARATIVE EVALUATION OF THE EFFICACY OF OCCLUSAL SPLINTS FABRICATED IN CENTRIC RELATION OR MAXIMUM INTERCUSPATION IN TEMPOROMANDIBULAR DISORDERS PATIENTS
}

\author{
Marcelo Matida HAMATA ${ }^{1}$, Paulo Renato Junqueira ZUIM ${ }^{2}$, Alicio Rosalino GARCIA ${ }^{2}$
}

1- DDS, MSc, Graduate Student, Department of Dental Materials and Prosthodontics, Dental School of Araçatuba, São Paulo State University, Araçatuba, SP, Brazil.

2- DDS, MSc, PhD, Assistant Professor, Department of Dental Materials and Prosthodontics, Dental School of Araçatuba, São Paulo State University, Araçatuba, SP, Brazil.

Corresponding address: Prof. Dr. Marcelo Matida Hamata - Departmento d eMateriais Dentários e Prótese - Faculdade de Odontologia de Araçatuba/ UNESP - Rua José Bonifácio, 1193 - 16015-050 - Araçatuba, SP - Brasil - Phone/Fax: +55-18-3636-3245 - e-mail: marcelopapa@yahoo.com; prjzuim@foa.unesp.br

Received: April 12, 2008 - Modification: July 10, 2008 - Accepted: August 30, 2008

\begin{abstract}
$\boldsymbol{H}$ abrication of occlusal splints in centric relation for temporomandibular disorders (TMD) patients is arguable, since this position has been defined for asymptomatic stomatognathic system. Thus, maximum intercuspation might be employed in patients with occlusal stability, eliminating the need for interocclusal records. This study compared occlusal splints fabricated in centric relation and maximum intercuspation in muscle pain reduction of TMD patients. Twenty patients with TMD of myogenous origin and bruxism were divided into 2 groups treated with splints in maximum intercuspation (I) or centric relation (II). Clinical, electrognathographic and electromyographic examinations were performed before and 3 months after therapy. Data were analyzed by the Student's t test. Differences at $5 \%$ level of probability were considered statistically significant. There was a remarkable reduction in pain symptomatology, without statistically significant differences $(p>0.05)$ between the groups. There was mandibular repositioning during therapy, as demonstrated by the change in occlusal contacts on the splints. Electrognathographic examination demonstrated a significant increase in maximum left lateral movement for group I and right lateral movement for group II ( $<<0.05$ ). There were no significant differences $(\mathrm{p}>0.05)$ in the electromyographic activities at rest after utilization of both splints. In conclusion, both occlusal splints were effective for pain control and presented similar action. The results suggest that maximum intercuspation may be used for fabrication of occlusal splints in patients with occlusal stability without large discrepancies between centric relation and maximum intercuspation. Moreover, this technique is simpler and less expensive.
\end{abstract}

Key words: Occlusal splints. Temporomandibular joint disorders. Centric relation.

\section{INTRODUCTION}

The influence of optimal occlusion provided by the occlusal splint on temporomandibular disorders (TMD) has been widely discussed ${ }^{10,11,17,20,24,26}$. Historically, the centric relation $(\mathrm{CR})$ has been advocated as a reference for fabrication of occlusal splints. Centric relation is currently understood as a maxillomandibular relationship in which the condyles articulate with the thinnest avascular portion of their respective disks with the complex in the anteriorsuperior position against the slopes of the articular eminences ${ }^{16}$. However, in symptomatic patients, utilization of the CR is arguable, since the CR has been defined for asymptomatic stomatognathic system. In the case of TMD, the joints and/or muscles are affected. The presence of pain, emotional or physical distress, inability of the operator and lack of neuromuscular conditioning may also influence the record of $\mathrm{CR}^{5,15}$.

Moreover, studies have demonstrated mandibular

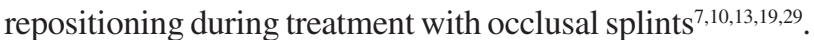
The final mandibular position (neuromuscular position) after occlusal adjustment of the splints at the follow-up sessions differs from the position at treatment onset (CR). This repositioning to the neuromuscular position is followed by a reduction in signs and symptoms of $\mathrm{TMD}^{7,10,13,19,29}$. According to Tripodakis, et al. ${ }^{30}$, the neuromuscular position is located between maximum intercuspation (MI) and CR in anteroposterior direction, without differences in laterolateral direction. Thus, the MI position may be a viable option as a starting point to the position of neuromuscular 
balance because it is easier to achieve and reduces the treatment costs and chair time.

The aim of this study was to comparatively evaluate the efficacy of occlusal splints fabricated in MI and CR, in patients with TMD of myogenous origin and bruxism.

\section{MATERIAL AND METHODS}

\section{Patients}

For the study, 20 TMD patients were selected from patients who came for treatment at the Temporomandibular Disorders Diagnostic and Treatment Center of the Dental School of Araçatuba, São Paulo State University, Brazil).

Patients with bruxism and TMD of myogenous origin were selected according to the research diagnostic criteria (RDC) for temporomandibular disorders ${ }^{9}$ and following specific inclusion criteria: a) patient's awareness of the bruxism; b) presence of signs and symptoms of TMD, characterizing localized muscle pain; c) Angle Class I molar relationship. The following exclusion criteria were adopted: a) dental treatment up to three months before therapy; b) any systemic pathology; c) use of removable dentures; e) more than one absent tooth per quadrant; f) patients scheduled for dental procedures that could alter the occlusion during therapy; g) occlusal interferences during excursive mandibular movements; h) TMD of articular origin.

The patients were randomly divided into 2 groups of 10 subjects each: Group I was treated with occlusal splints in MI, and Group II was treated with occlusal splints fabricated from the CR record.

All procedures of this study and the selection of patients were performed by the same examiner.

\section{Occlusal Splints}

Impressions of the maxillary and mandibular dental arches of patients were taken with irreversible hydrocolloid (Hydrogum, Zhermack S.p.A., Badia Polesine, Italy) and poured with special plaster (Durone IV; Dentsply Indústria e Comércio LTDA, Rio de Janeiro, Brazil). The maxillary dental casts were mounted in an articulator with aid of the face bow. The difference between the 2 splints was the intermaxillary positioning of mounting of mandibular casts in the articulator, either MI or CR.

The occlusal splints were fabricated with acrylic resin (Clássico; Artigos Odontológicos Clássico LTDA, São Paulo, SP, Brazil), completely covering the maxillary teeth, with approximate thickness of $2 \mathrm{~mm}$ in the molar region, simultaneous bilateral occlusal contacts and immediate disocclusion of the posterior teeth by the anterior teeth during excursive mandibular movements ${ }^{2,6,7,10-12,19,20,22,23,25,26,31}$.

Initially, the splints were adjusted as to the adaptation and comfort. Occlusal adjustments were performed with the patient in supine position, following the free mandibular closure secondary to neuromuscular balance $e^{3,8,13}$. No polishing was performed after adjustment for maintenance of the refined occlusal contacts. ${ }^{2}$

The patients were instructed to wear the occlusal splint at nighttime and were informed on the cyclic nature of TMD and the relationship between parafunction, muscle fatigue, muscle pain and psychological factors ${ }^{11,14,28}$. In an attempt to alleviate daytime harmful habits, the patients were instructed to seal the lips and separate the teeth, keeping the muscles relaxed ${ }^{26}$.

Occlusal splints in MI: These splints were fabricated by mounting the mandibular cast in the articulator by tactile and visual intercuspation. After mounting, the upper arm of the articulator was lifted in $3 \mathrm{~mm}$ to allow enough interocclusal space for fabrication of the splint with the desired thickness ( $2 \mathrm{~mm})$.

Occlusal splints in CR: These splints were fabricated after mounting the mandibular cast in the articulator with aid of wax (Wilson; Polidental Indústria e Comércio LTDA, Cotia, Brazil) and acrylic resin (Duralay; Reliance Dental Mfg Co. Wort, IL, USA) bite record, similar to the technique described by Bezzon and Orsi ${ }^{4}$. The record is obtained in a two-step procedure. In the first step, the pre-trimmed wax is placed in the mouth and the patient closes the mouth in CR guided by an anterior acrylic resin device (Lucia's jig) ${ }^{21}$ and by the bimanual manipulation of Dawson. The second step comprised perforation of the wax at four points on the indentations at the region of first premolars and second molars at both sides, followed by refinement of the record with acrylic resin Duralay in CR position. The casts were then mounted with aid of a rigid and stable material, with lower possibility of deformation or distortion. The incisal pin of the articulator was kept at position " 0 " and the splint thickness $(2 \mathrm{~mm})$ was established by the record thickness.

\section{Clinical Examination}

Pain symptomatology: The intensity, duration (hours per day) and frequency (days per week) of pain symptoms and the intensity of pain to palpation of the masseter and temporalis muscles were evaluated. The period of pain (months) was also recorded. ${ }^{11}$ The intensity of pain symptoms and pain to palpation was evaluated with aid of a visual analogue scale from " 0 " to " 10 ", in which " 0 " represented no pain and " 10 " the worst pain ever felt by the patient ${ }^{11,13,14,20,28}$. Palpation was performed, bilaterally and separately, at the following regions: origin, body and insertion of the masseter muscle and anterior, medium and posterior portions of the temporalis muscle ${ }^{11}$. A digital pressure of about $2 \mathrm{Kgf}$ was used for palpation. A mean was calculated for each muscle by adding the number relative to the pain of each palpated region and division of this value by 3 . After 3 months, the pain symptomatology was reevaluated by the same method, for evaluation of the percentage of reduction in frequency, duration and intensity of pain symptoms and pain to palpation compared to the onset of therapy between groups. The percentage of reduction was employed to avoid influence of subjectivity of perception of pain on the results.

Clinical observations: The period required for occlusal stabilization on the splints representing neuromuscular balance and the reports of patients ${ }^{27}$ were observed. After placement of the splints, the patients were followed-up for 
3 months, weekly in the first month and monthly in the following months. At the 6 follow-up visits, the occlusal contacts on the splints were checked and adjustments were performed in the absence of one or more teeth in contact. Occlusal stability was considered when no change was observed at 2 consecutive follow-up visits ${ }^{26}$.

\section{Electrognathographic Examination}

Electrognathography was conducted to evaluate the amplitude of mandibular movement (maximum mouth opening, maximum protrusion and maximum right and left lateral movement) before and 3 months after therapy. A magneto was fixated to the region of mandibular central incisors with sticky wax, parallel to the bipupillar line. A sensor was positioned on the patients' heads parallel to the magnet and recorded the pathway and extent of movements in millimeters. The information was transmitted to a computer and analyzed as graphs using the software Bio EGN (System; Bio-Research Associates, Inc., Milwaukee, WI, USA,). During examination, the patients were kept seated and straight and were asked to look at the horizon. All records were initiated in MI.

\section{Electromyographic Examination}

Electromyographic examination of the masseter and anterior temporalis muscles ${ }^{1,18}$ at both sides was performed before and 3 months after therapy, without wearing the splint. For that purpose, the skin at these regions and neck was cleaned with soap and water followed by a gauze soaked in alcohol. Next, surface electrodes (Medi-Trace 200; Kendall LTP, Chicopee, USA) were placed, following the long axis of muscle fibers of the evaluated muscles, and at the neck region (ground wire). Information on the muscle activity was transferred to a computer and analyzed in microvolts $(\mu \mathrm{V})$ with aid of the software Bio EMG (System; BioResearch, INC, Milwaukee, USA). The records were obtained at rest, with the patients seated in orthostatic position, looking at the horizon and keeping their teeth slightly separated.

TABLE 1- Percentage of reduction in intensity, frequency and duration of pain symptoms and period of pain in groups I (MI) and II (CR), and initial and final values of pain intensity, frequency and duration in parentheses

\begin{tabular}{|c|c|c|c|c|c|c|c|c|}
\hline \multirow[t]{2}{*}{ Pat. } & \multicolumn{2}{|c|}{$\begin{array}{c}\text { Intensity } \\
\% R(V A S / 0-10)\end{array}$} & \multicolumn{2}{|c|}{$\begin{array}{c}\text { Frequency } \\
\% R \text { (days/week) }\end{array}$} & \multicolumn{2}{|c|}{$\begin{array}{c}\text { Duration } \\
\% R \text { (hours/day) }\end{array}$} & \multicolumn{2}{|c|}{$\begin{array}{c}\text { Period } \\
\text { (months) }\end{array}$} \\
\hline & MI & CR & MI & CR & MI & CR & MI & CR \\
\hline 1 & $\begin{array}{l}100 \\
(2-0)\end{array}$ & $\begin{array}{l}100 \\
(5-0)\end{array}$ & $\begin{array}{l}100 \\
(3-0)\end{array}$ & $\begin{array}{l}57.1 \\
(7-3)\end{array}$ & $\begin{array}{c}100 \\
(16-0)\end{array}$ & $\begin{array}{l}37.5 \\
(8-5)\end{array}$ & 36 & 2 \\
\hline 2 & $\begin{array}{l}100 \\
(2-0)\end{array}$ & $\begin{array}{l}100 \\
(3-0)\end{array}$ & $\begin{array}{c}75 \\
(4-1)\end{array}$ & $\begin{array}{l}100 \\
(2-0)\end{array}$ & $\begin{array}{c}80 \\
(5-1)\end{array}$ & $\begin{array}{l}100 \\
(8-0)\end{array}$ & 18 & 72 \\
\hline 3 & $\begin{array}{l}100 \\
(7-0)\end{array}$ & $\begin{array}{l}100 \\
(5-0)\end{array}$ & $\begin{array}{l}100 \\
(4-0)\end{array}$ & $\begin{array}{c}75 \\
(4-1)\end{array}$ & $\begin{array}{c}100 \\
(16-0)\end{array}$ & $\begin{array}{c}96.8 \\
(16-0.5)\end{array}$ & 18 & 24 \\
\hline 4 & $\begin{array}{l}100 \\
(8-0)\end{array}$ & $\begin{array}{l}100 \\
(6-0)\end{array}$ & $\begin{array}{l}100 \\
(7-0)\end{array}$ & $\begin{array}{l}100 \\
(3-0)\end{array}$ & $\begin{array}{c}100 \\
(16-0)\end{array}$ & $\begin{array}{c}100 \\
(16-0)\end{array}$ & 6 & 24 \\
\hline 5 & $\begin{array}{r}100 \\
(6-0)\end{array}$ & $\begin{array}{l}100 \\
(2-0)\end{array}$ & $\begin{array}{c}80 \\
(5-1)\end{array}$ & $\begin{array}{l}100 \\
(7-0)\end{array}$ & $\begin{array}{c}50 \\
(6-3)\end{array}$ & $\begin{array}{l}100 \\
(8-0)\end{array}$ & 36 & 18 \\
\hline 6 & $\begin{array}{l}100 \\
(4-0)\end{array}$ & $\begin{array}{l}100 \\
(2-0)\end{array}$ & $\begin{array}{c}80 \\
(5-1)\end{array}$ & $\begin{array}{l}33.3 \\
(3-2)\end{array}$ & $\begin{array}{c}50 \\
(2-1)\end{array}$ & $\begin{array}{c}50 \\
(1-0.5)\end{array}$ & 36 & 12 \\
\hline 7 & $\begin{array}{l}100 \\
(8-0)\end{array}$ & $\begin{array}{l}100 \\
(6-0)\end{array}$ & $\begin{array}{l}100 \\
(7-0)\end{array}$ & $\begin{array}{l}100 \\
(7-0)\end{array}$ & $\begin{array}{c}100 \\
(16-0)\end{array}$ & $\begin{array}{c}100 \\
(16-0)\end{array}$ & 12 & 8 \\
\hline 8 & $\begin{array}{l}100 \\
(6-0)\end{array}$ & $\begin{array}{l}100 \\
(7-0)\end{array}$ & $\begin{array}{l}85.7 \\
(7-1)\end{array}$ & $\begin{array}{l}100 \\
(7-0)\end{array}$ & $\begin{array}{c}50 \\
(4-2)\end{array}$ & $\begin{array}{c}100 \\
(16-0)\end{array}$ & 6 & 48 \\
\hline 9 & $\begin{array}{r}100 \\
(4-0)\end{array}$ & $\begin{array}{l}85.7 \\
(7-1)\end{array}$ & $\begin{array}{l}100 \\
(6-0)\end{array}$ & $\begin{array}{c}50 \\
(4-2)\end{array}$ & $\begin{array}{l}100 \\
(5-0)\end{array}$ & $\begin{array}{l}87.5 \\
(16-2)\end{array}$ & 4 & 8 \\
\hline 10 & $\begin{array}{l}100 \\
(2-0)\end{array}$ & $\begin{array}{l}33.3 \\
(3-2)\end{array}$ & $\begin{array}{l}100 \\
(2-0)\end{array}$ & $\begin{array}{c}75 \\
(4-1)\end{array}$ & $\begin{array}{l}100 \\
(2-0)\end{array}$ & $\begin{array}{c}60 \\
(5-2)\end{array}$ & 6 & 36 \\
\hline Mean & $\begin{array}{c}100 \\
(4.9-0)\end{array}$ & $\begin{array}{c}91.9 \\
(4.6-0.3)\end{array}$ & $\begin{array}{c}92 \\
(5-0.4)\end{array}$ & $\begin{array}{c}79 \\
(4.8-0.8)\end{array}$ & $\begin{array}{c}83 \\
(8.8-0.7)\end{array}$ & $\begin{array}{l}83.1 \\
(11-1)\end{array}$ & 17.8 & 25.2 \\
\hline SD & - & 21 & 10.5 & 25 & 23.5 & 24.3 & 13.4 & 21.5 \\
\hline $\begin{array}{c}\text { t-test } \\
\text { df=18 } \\
P\end{array}$ & \multicolumn{2}{|c|}{1.22} & \multicolumn{2}{|c|}{1.52} & \multicolumn{2}{|c|}{0.02} & \multicolumn{2}{|c|}{-} \\
\hline
\end{tabular}




\section{Statistical Analysis}

Electrognathography and electromyography were analyzed by the Student's t test for paired samples, comparing the data achieved before and after treatment (intragroup analysis). Data related to the degree (\%) of reduction of pain symptomatology were analyzed by the Student's $t$ test for independent samples for observation of differences between groups (intergroup analysis). Differences at the $5 \%$ level of probability were considered statistically significant.

\section{RESULTS}

\section{Patients}

There was predominance of females in group I ( 3 men and 7 women) and group II ( 1 men and 9 women) and similar mean age for both groups. Mean age of group I and II was, respectively, 23.9 (13-39) and 23.8 (17-40).

\section{Clinical Examination}

Pain symptomatology: There was a remarkable reduction in pain symptomatology in the 2 groups. There were no significant differences $(p<0.05)$ between groups in the evaluation of intensity, frequency and duration of pain symptoms and intensity of pain to palpation of the masseter and temporalis muscles at both sides. The period of existence of the symptoms varied for the entire sample. Tables 1 and 2 demonstrate these results and the initial and final values of intensity, frequency and duration of pain of all patients.

Clinical observations: a) Considering the occlusal stability on the splint if no change was required at 2 consecutive follow-up visits, it could be observed that, in group I, 4 patients presented stability at the second followup, 3 at the third, 1 at the fourth, 1 at the fifth and 1 at the sixth follow-up. In group II, 4 patients achieved stability at the second follow-up, 2 at the third, 2 at the fourth and 2 patients did not present occlusal stability. Moreover, it was observed that 3 patients in group I and 2 in group II presented

TABLE 2- Percentage of reduction in pain to palpation of the temporalis and masseter muscles at the right and left sides in groups I (MI) and II (CR), and initial and final values of pain to palpation in parentheses

\begin{tabular}{|c|c|c|c|c|c|c|c|c|}
\hline \multirow[t]{3}{*}{ Pat. } & \multicolumn{4}{|c|}{$\begin{array}{l}\text { Masseter } \\
\% \text { R (VAS) }\end{array}$} & \multicolumn{4}{|c|}{$\begin{array}{c}\text { Temporalis } \\
\text { \%R (VAS) }\end{array}$} \\
\hline & \multicolumn{2}{|c|}{ Right } & \multicolumn{2}{|c|}{ Left } & \multicolumn{2}{|c|}{ Right } & \multicolumn{2}{|c|}{ Left } \\
\hline & MI & CR & MI & CR & MI & CR & MI & CR \\
\hline 1 & $\begin{array}{c}84.3 \\
(8.3-1.3)\end{array}$ & $\begin{array}{c}72.2 \\
(8.3-2.3)\end{array}$ & $\begin{array}{c}78.3 \\
(6-1.3)\end{array}$ & $\begin{array}{c}72.2 \\
(8.3-2.3)\end{array}$ & $\begin{array}{l}100 \\
(5-0)\end{array}$ & $\begin{array}{c}83.1 \\
(7.7-1.3)\end{array}$ & $\begin{array}{c}94.7 \\
(5.7-0.3)\end{array}$ & $\begin{array}{c}68.2 \\
(6.3-2)\end{array}$ \\
\hline 2 & $\begin{array}{l}100 \\
(4-0)\end{array}$ & $\begin{array}{c}89.2 \\
(5.6-0.6)\end{array}$ & $\begin{array}{c}88.8 \\
(6.3-0.7)\end{array}$ & $\begin{array}{c}91 \\
(6.7-0.6)\end{array}$ & $\begin{array}{l}100 \\
(3-0)\end{array}$ & $\begin{array}{c}100 \\
(5.3-0)\end{array}$ & $\begin{array}{c}90 \\
(3-0.3)\end{array}$ & $\begin{array}{c}94.3 \\
(5.3-0.3)\end{array}$ \\
\hline 3 & $\begin{array}{c}63 \\
(7.3-2.7)\end{array}$ & $\begin{array}{c}94 \\
(5-0.3)\end{array}$ & $\begin{array}{c}49.3 \\
(7.3-3.7)\end{array}$ & $\begin{array}{c}93 \\
(4.3-0.3)\end{array}$ & $\begin{array}{c}100 \\
(2.7-0)\end{array}$ & $\begin{array}{l}100 \\
(4-0)\end{array}$ & $\begin{array}{l}100 \\
(3-0)\end{array}$ & $\begin{array}{c}100 \\
(4.7-0)\end{array}$ \\
\hline 4 & $\begin{array}{l}100 \\
(9-0)\end{array}$ & $\begin{array}{c}100 \\
(7-0)\end{array}$ & $\begin{array}{l}100 \\
(5-0)\end{array}$ & $\begin{array}{l}100 \\
(8-0)\end{array}$ & $\begin{array}{l}87.5 \\
(8-1)\end{array}$ & $\begin{array}{l}100 \\
(6-0)\end{array}$ & $\begin{array}{c}100 \\
(5.7-0)\end{array}$ & $\begin{array}{l}100 \\
(5-0)\end{array}$ \\
\hline 5 & $\begin{array}{c}80.5 \\
(6.7-1.3)\end{array}$ & $\begin{array}{c}72.2 \\
(8.3-2.3)\end{array}$ & $\begin{array}{c}77.1 \\
(5.7-1.3)\end{array}$ & $\begin{array}{c}47.6 \\
(6.3-3.3)\end{array}$ & $\begin{array}{c}93.6 \\
(4.7-0.3)\end{array}$ & $\begin{array}{l}100 \\
(2-0)\end{array}$ & $\begin{array}{c}94 \\
(5-0.3)\end{array}$ & $\begin{array}{l}100 \\
(3-0)\end{array}$ \\
\hline 6 & $\begin{array}{c}77.9 \\
(7.7-1.7)\end{array}$ & $\begin{array}{c}57.1 \\
(6.3-2.7)\end{array}$ & $\begin{array}{c}85 \\
(6.7-1)\end{array}$ & $\begin{array}{c}54 \\
(5-2.3)\end{array}$ & $\begin{array}{c}100 \\
(0.3-0)\end{array}$ & $\begin{array}{c}74 \\
(2.7-0.7)\end{array}$ & $\begin{array}{l}100 \\
(2-0)\end{array}$ & $\begin{array}{c}43.3 \\
(3-1.7)\end{array}$ \\
\hline 7 & $\begin{array}{c}100 \\
(8.7-0)\end{array}$ & $\begin{array}{l}100 \\
(6-0)\end{array}$ & $\begin{array}{l}100 \\
(8-0)\end{array}$ & $\begin{array}{c}100 \\
(5.3-0)\end{array}$ & $\begin{array}{c}100 \\
(7.3-0)\end{array}$ & $\begin{array}{l}100 \\
(1-0)\end{array}$ & $\begin{array}{l}100 \\
(8-0)\end{array}$ & $\begin{array}{l}100 \\
(5-0)\end{array}$ \\
\hline 8 & $\begin{array}{l}71.4 \\
(7-2)\end{array}$ & $\begin{array}{c}60 \\
(5-2)\end{array}$ & $\begin{array}{l}71.4 \\
(7-2)\end{array}$ & $\begin{array}{c}61.1 \\
(6.7-2.6)\end{array}$ & $\begin{array}{c}88.3 \\
(6-0.7)\end{array}$ & $\begin{array}{c}70 \\
(1-0.3)\end{array}$ & $\begin{array}{l}83.3 \\
(6-1)\end{array}$ & $\begin{array}{c}76.9 \\
(1.3-0.3)\end{array}$ \\
\hline 9 & $\begin{array}{c}89.6 \\
(9.7-1)\end{array}$ & $\begin{array}{c}84.1 \\
(6.3-1)\end{array}$ & $\begin{array}{c}100 \\
(8.7-0)\end{array}$ & $\begin{array}{c}87.7 \\
(5.7-0.7)\end{array}$ & $\begin{array}{c}100 \\
(8.3-0)\end{array}$ & $\begin{array}{l}100 \\
(5-0)\end{array}$ & $\begin{array}{l}100 \\
(8-0)\end{array}$ & $\begin{array}{l}100 \\
(5-0)\end{array}$ \\
\hline 10 & $\begin{array}{c}100 \\
(3.7-0)\end{array}$ & $\begin{array}{c}55.2 \\
(6.7-3)\end{array}$ & $\begin{array}{c}100 \\
(3.3-0)\end{array}$ & $\begin{array}{c}74 \\
(7.7-2)\end{array}$ & $\begin{array}{c}100 \\
(0.3-0)\end{array}$ & $\begin{array}{c}87.7 \\
(5.7-0.7\end{array}$ & $\begin{array}{c}100 \\
(0.6-0)\end{array}$ & $\begin{array}{c}67.9 \\
(5.3-1.7)\end{array}$ \\
\hline Mean & $\begin{array}{c}86.6 \\
(7.2-1)\end{array}$ & $\begin{array}{c}78.4 \\
(6.4-1.4)\end{array}$ & $\begin{array}{c}84.9 \\
(6.4-1)\end{array}$ & $\begin{array}{c}78 \\
(6.3-1.4)\end{array}$ & $\begin{array}{c}96.9 \\
(4.5-0.2)\end{array}$ & $\begin{array}{c}91.4 \\
(4-0.3)\end{array}$ & $\begin{array}{c}96.2 \\
(4.7-0.2)\end{array}$ & $\begin{array}{c}85 \\
(5.3-1.7)\end{array}$ \\
\hline SD & 13,4 & 17.4 & 16.6 & 19.1 & 5.1 & 11.9 & 5.7 & 19.9 \\
\hline $\begin{array}{l}t \text { test } \\
d t=18\end{array}$ & \multicolumn{2}{|c|}{1.19} & \multicolumn{2}{|c|}{0.87} & \multicolumn{2}{|c|}{1.33} & \multicolumn{2}{|c|}{1.69} \\
\hline$P$ & \multicolumn{2}{|c|}{0.2494 (ns) } & \multicolumn{2}{|c|}{$0.5974(\mathrm{~ns})$} & \multicolumn{2}{|c|}{$0.1992(\mathrm{~ns})$} & \multicolumn{2}{|c|}{$0.1042(\mathrm{~ns})$} \\
\hline
\end{tabular}

Pat., patient; \%R, percentage of reduction; VAS, visual analogue scale; $M I$, maximum intercuspation; $C R=$ centric relation; $S D$, standard deviation; $d f$, degrees of freedom; $P$, probability; $n s$, non-significant. 
TABLE 3- Amplitude of mandibular movements before and after treatment of patients in group I (MI) and group II (CR)

\begin{tabular}{|c|c|c|c|c|c|c|c|c|c|c|c|c|c|c|c|c|}
\hline \multirow{3}{*}{ Pat. } & \multicolumn{4}{|c|}{ MMO } & \multicolumn{4}{|c|}{ Protrusion } & \multicolumn{4}{|c|}{ RLM } & \multicolumn{4}{|c|}{ LLM } \\
\hline & \multicolumn{2}{|c|}{ MI } & \multicolumn{2}{|c|}{ CR } & \multicolumn{2}{|c|}{ MI } & \multicolumn{2}{|c|}{ CR } & \multicolumn{2}{|c|}{ MI } & \multicolumn{2}{|c|}{ CR } & \multicolumn{2}{|c|}{ MI } & \multicolumn{2}{|c|}{ CR } \\
\hline & B & A & B & A & B & A & B & A & B & A & B & A & B & A & B & A \\
\hline 1 & 46.4 & 54.6 & 51.7 & 48.8 & 9.1 & 8.1 & 4.7 & 5.6 & 10.6 & 10.3 & 7.8 & 8.1 & 10 & 9.7 & 5.6 & 6.2 \\
\hline 2 & 49.5 & 45.8 & 46.2 & 55.7 & 7.8 & 8.4 & 5.3 & 5.3 & 9.7 & 10.3 & 7.2 & 7.8 & 7.8 & 10.3 & 7.8 & 6.9 \\
\hline 3 & 47.5 & 49.9 & 52 & 48.3 & 5.3 & 7.8 & 7.5 & 8.8 & 8.8 & 5.9 & 6.2 & 8.4 & 6.9 & 7.5 & 7.5 & 9.4 \\
\hline 4 & 51.3 & 50.1 & 48.6 & 55.2 & 5.9 & 7.8 & 5.6 & 5.6 & 4.1 & 3.8 & 6.9 & 8.4 & 3.4 & 5.0 & 6.9 & 8.8 \\
\hline 5 & 43.6 & 44.3 & 40.7 & 40.6 & 4.7 & 6.9 & 6.6 & 5.6 & 3.1 & 5.9 & 3.8 & 8.8 & 4.1 & 7.8 & 7.2 & 6.2 \\
\hline 6 & 51.3 & 46 & 47.7 & 41.7 & 8.8 & 8.8 & 7.5 & 6.2 & 5.9 & 9.4 & 9.7 & 9.4 & 7.8 & 9.1 & 10 & 9.1 \\
\hline 7 & 46.2 & 49.3 & 48.6 & 49.1 & 9.4 & 8.1 & 9.7 & 6.9 & 9.4 & 8.1 & 7.2 & 16.2 & 10 & 10 & 8.8 & 15.3 \\
\hline 8 & 57.1 & 56.5 & 47.3 & 51.7 & 5.9 & 6.2 & 4.1 & 5.6 & 7.2 & 6.2 & 6.9 & 9.4 & 8.8 & 9.7 & 7.8 & 7.8 \\
\hline 9 & 45 & 46.7 & 49.1 & 51.8 & 9.1 & 8.1 & 7.2 & 7.5 & 10 & 9.7 & 9.4 & 10.3 & 10 & 8.8 & 9.1 & 10.3 \\
\hline 10 & 50.4 & 48.1 & 47.7 & 49.8 & 8.1 & 9.4 & 8.1 & 6.9 & 7.8 & 10 & 6.9 & 7.8 & 6.9 & 9.1 & 8.8 & 9.4 \\
\hline $\begin{array}{c}\text { Mean } \\
\text { SD }\end{array}$ & $\begin{array}{c}48.8 \\
3.9\end{array}$ & $\begin{array}{c}49.1 \\
3.9\end{array}$ & $\begin{array}{c}47.9 \\
3.1\end{array}$ & $\begin{array}{c}49.2 \\
4.9\end{array}$ & $\begin{array}{l}7.4 \\
1.7\end{array}$ & $\begin{array}{l}7.9 \\
0.9\end{array}$ & $\begin{array}{l}6.6 \\
1.7\end{array}$ & $\begin{array}{l}6.4 \\
1.1\end{array}$ & $\begin{array}{l}7.6 \\
2.5\end{array}$ & $\begin{array}{l}7.9 \\
2.3\end{array}$ & $\begin{array}{l}7.2 \\
1.6\end{array}$ & $\begin{array}{l}9.4 \\
2.4\end{array}$ & $\begin{array}{l}7.5 \\
2.3\end{array}$ & $\begin{array}{l}8.7 \\
1.5\end{array}$ & $\begin{array}{l}7.9 \\
1.2\end{array}$ & $\begin{array}{l}8.9 \\
2.6\end{array}$ \\
\hline t-test & 0 & & 0.8 & 85 & 1. & 25 & & 54 & 0 & 48 & & 55 & & .46 & & 41 \\
\hline $\begin{array}{c}\mathrm{df}=9 \\
P\end{array}$ & & & $\begin{array}{r}0.5 \\
(\mathrm{n}\end{array}$ & $\begin{array}{l}780 \\
\text { s) }\end{array}$ & & $\begin{array}{l}428 \\
\text { s) }\end{array}$ & & $\begin{array}{l}064 \\
\text { s) }\end{array}$ & & & & $298^{*}$ & & $346^{*}$ & & $\begin{array}{l}906 \\
\text { is) }\end{array}$ \\
\hline
\end{tabular}

Pat., patient; $M M O$, maximum mouth opening; $R L M$, right lateral movement; $L L M$, left lateral movement, $B$, before; $A$, after; $S D$, standard deviation; $d f$, degrees of freedom; $P$, probability; $n s$, non-significant; ${ }^{*}$, statistically significant at $5 \%$.

TABLE 4- Electromyographic activity in $\mu \mathrm{V}$ of the masseter and anterior temporalis muscles for group I (MI) e group II (CR) at rest, before and after treatment

\begin{tabular}{|c|c|c|c|c|c|c|c|c|c|c|c|c|c|c|c|c|}
\hline \multirow{3}{*}{ Pat. } & \multicolumn{4}{|c|}{ MMO } & \multicolumn{4}{|c|}{ Protrusion } & \multicolumn{4}{|c|}{ RLM } & \multicolumn{4}{|c|}{ LLM } \\
\hline & \multicolumn{2}{|c|}{ MI } & \multicolumn{2}{|c|}{ CR } & \multicolumn{2}{|c|}{ MI } & \multicolumn{2}{|c|}{ CR } & \multicolumn{2}{|c|}{ MI } & \multicolumn{2}{|c|}{ CR } & \multicolumn{2}{|c|}{ MI } & \multicolumn{2}{|c|}{ CR } \\
\hline & B & A & B & A & B & A & B & $A$ & B & A & B & $A$ & B & A & B & A \\
\hline 1 & 2.2 & 1.8 & 1.2 & 2.0 & 1.3 & 2.1 & 1.2 & 1.3 & 2.0 & 2.7 & 2.2 & 2.4 & 1.9 & 2.1 & 1.8 & 2.7 \\
\hline 2 & 1.6 & 1.0 & 1.3 & 1.1 & 1.3 & 1.4 & 1.0 & 1.3 & 2.2 & 2.3 & 3.0 & 2.3 & 1.3 & 1.4 & 1.6 & 2.1 \\
\hline 3 & 1.4 & 1.4 & 3.1 & 1.2 & 2.2 & 2.0 & 1.2 & 1.2 & 1.9 & 1.4 & 2.6 & 1.5 & 2.1 & 1.6 & 1.4 & 2.0 \\
\hline 4 & 1.2 & 1.2 & 1.2 & 1.3 & 1.0 & 0.9 & 1.4 & 1.6 & 1.6 & 3.2 & 1.7 & 1.6 & 2.0 & 2.8 & 3.9 & 2.6 \\
\hline 5 & 1.3 & 1.8 & 3.3 & 1.8 & 1.4 & 2.9 & 2.0 & 1.2 & 1.6 & 2.2 & 2.2 & 2.0 & 1.7 & 2.3 & 1.8 & 1.3 \\
\hline 6 & 1.1 & 1.5 & 2.0 & 1.4 & 1.3 & 1.7 & 1.3 & 1.1 & 1.7 & 1.9 & 1.8 & 1.2 & 1.0 & 1.3 & 1.4 & 1.4 \\
\hline 7 & 1.4 & 1.8 & 1.2 & 1.1 & 1.1 & 1.5 & 1.2 & 1.4 & 2.2 & 1.5 & 1.0 & 1.6 & 1.2 & 1.3 & 1.1 & 1.2 \\
\hline 8 & 2.2 & 1.5 & 1.8 & 1.6 & 1.8 & 1.3 & 1.4 & 2.5 & 1.7 & 1.1 & 1.8 & 2.1 & 2.6 & 2.7 & 1.8 & 1.2 \\
\hline 9 & 1.8 & 1.3 & 3.7 & 1.5 & 2.2 & 1.0 & 2.0 & 1.4 & 1.9 & 1.3 & 2.6 & 1.6 & 1.2 & 1.8 & 1.6 & 1.3 \\
\hline 10 & 1.7 & 1.4 & 1.2 & 1.1 & 1.3 & 1.1 & 1.4 & 1.2 & 2.0 & 1.9 & 1.5 & 1.8 & 1.7 & 1.2 & 1.2 & 1.3 \\
\hline $\begin{array}{l}\text { Mean } \\
\text { SD }\end{array}$ & $\begin{array}{l}1.5 \\
0.3\end{array}$ & $\begin{array}{l}1.4 \\
0.2\end{array}$ & $\begin{array}{l}2.0 \\
0.9\end{array}$ & $\begin{array}{l}1.4 \\
0.3\end{array}$ & $\begin{array}{l}1.4 \\
0.4\end{array}$ & $\begin{array}{l}1.5 \\
0.6\end{array}$ & $\begin{array}{l}1.4 \\
0.3\end{array}$ & $\begin{array}{l}1.4 \\
0.4\end{array}$ & $\begin{array}{l}1.8 \\
0.2\end{array}$ & $\begin{array}{l}1.9 \\
0.6\end{array}$ & $\begin{array}{l}2.0 \\
0.5\end{array}$ & $\begin{array}{l}1.8 \\
0.3\end{array}$ & $\begin{array}{l}1.6 \\
0.4\end{array}$ & $\begin{array}{l}1.8 \\
0.5\end{array}$ & $\begin{array}{l}1.7 \\
0.7\end{array}$ & $\begin{array}{l}1.7 \\
0.5\end{array}$ \\
\hline t-test & & & & & & & & 06 & & & & & & & & \\
\hline$P$ & & & & & & & & $\begin{array}{l}082 \\
\text { s) }\end{array}$ & & & & & & & & \\
\hline
\end{tabular}

Pat., patient; $B$, before; $A$, after; $S D$, standard deviation; $d f$, degrees of freedom; $P$, probability; ns, non-significant. 
instability of contacts after achievement of stability at two consecutive follow-up visits. b) During treatment, the patients in both groups reported some experiences: 1) occlusion on the splint had been changed and the contacts established at previous follow-up visits were no longer present, especially at the anterior region of the splint; 2) after removal of the splint at morning, there was difficulty to achieve occlusion in MI; 3) the splint increased the awareness of parafunction.

\section{Electrognathographic Examination}

Group I presented increased amplitude of all movements, yet with statistically significant difference $(\mathrm{p}<0.05)$ only at maximum left lateral movement. In group II, there was a reduction in amplitude in maximum protrusion and increase in the other movements. However, statistically significant difference $(\mathrm{p}<0.05)$ was only observed at maximum right lateral movement (Table 3).

\section{Electromyographic Evaluation}

There were no significant changes $(\mathrm{p}<0.05)$ in the electromyographic activities of the masseter and anterior temporalis muscle at 3 months after utilization of splints in both groups (Table 4).

\section{DISCUSSION}

Clinical studies have demonstrated mandibular repositioning after splint therapy. This repositioning to the neuromuscular position, different from the initial CR, is followed by a reduction in the signs and symptoms of TMD and seems to be a more physiological and balanced position for the muscles ${ }^{7,10,13,19,29}$. It is difficult to determine when the patient achieves neuromuscular balance on the splint and adjustments are no longer required. In the study of Rubinoff, et $\mathrm{al}^{26}$, the adjustments were continued until no change in contacts was observed at 2 consecutive follow-up visits. In some patients of the present study ( 3 in group I and 2 in group II), this criterion was not very valuable, since there was fluctuation of stability of contacts on the splint, even after achievement of stability at two consecutive follow-up visits. Moreover, 2 patients in group II did not achieve occlusal stability according to the requirements of Rubinoff, et al. ${ }^{26}$. These findings may be explained by two reasons. The first would be related to the normal variation in the arc of closure, which occurs during the day. According to Berry and $\mathrm{Singh}^{3}$, this depends on the physical status of the muscles, which may be influenced by physical and mental distress. The second would be the presence of pain on palpation, which was not completely reduced in these patients. This demonstrates some change in the physical status of muscles, which might lead to instability in the arc of mandibular closure.

With regard to the improvement of pain symptomatology, there were no differences between groups. Moreover, the patients in both groups reported some aspects: 1) occlusion on the splint had been changed, with absence of contacts, especially at the anterior region; 2) after removal of the splint at morning, there was difficulty to achieve occlusion in MI; 3 ) the splint increased the awareness of parafunction. Reports 1 and 2 may be explained by the theory of maxillomandibular realignment, since mandibular repositioning was observed, as demonstrated by the change in occlusal contacts on the splint at the follow-up visits ${ }^{7,10,13,19,27}$. These findings suggest that, in asymptomatic patients, neither MI nor CR are positions of neuromuscular balance, when the mandible is positioned more physiologically. The absence of anterior contacts on the splint at the follow-up visits suggests mandibular retrusion, which may have occurred due to relaxation of the inferior lateral pterygoid muscles, which are responsible for anterior condylar movement. The difficulty of occlusion demonstrates the difference between the arc of closure in MI and the arc of closure in the neuromuscular balanced position.

The increased awareness of parafunction may be explained by the theory of cognitive perception. Occlusal coverage and the dental contacts on the splint seem to be important factors that, combined to the change in tongue positioning, increase the patient's awareness ${ }^{24,25}$.

With regard to electrognathography, there were no significant changes in maximum mouth opening and protrusion after therapy for both groups. However, there was a significant increase in maximum left lateral movement in group I and maximum right lateral movement in group II. Even though the amplitude of mandibular movements is important for the diagnosis and evaluation of TMD treatment, the initial extent of these movements did not pose a limitation.

Many studies have demonstrated the efficacy of occlusal splint to reduce the electromyographic activity at rest, maximum clenching and during nighttime parafunctional activity ${ }^{1,8,12,18,19,25}$. In the present study, this activity was evaluated without utilization of the occlusal splint and at rest. There were no significant changes in the electromyographic activity after therapy in both groups. This fact demonstrates that, if the splint reduces the electromyographic activity, this reduction does not seem to be permanent.

The results achieved enable fabrication of occlusal splints in MI. However, it should be mentioned that the patients selected in this study did not present large differences between CR and MIH. Besides the occlusal stability required for intercuspation of maxillary and mandibular dental casts, the difference between CR and MI should be considered. When there are large differences between these positions, use of CR may be more favorable. For example, a patient with pseudo Class III resulting from a large difference between CR and MI may present occlusal stability. However, the condyle may be improperly positioned, at the crest of the articular eminence, and the mandible may be very distant from the neuromuscular balance position. 


\section{CONCLUSION}

Based on the methodology, study sample and results obtained in the present study, it was concluded that both types of splint were effective and presented similar performance for all variables investigated.

\section{REFERENCES}

1- Al Quran FAM, Lyons MF. The immediate effect of hard and soft splints on the EMG activity of the masseter and temporalis muscles. J Oral Rehabil. 1999;26(7):559-63.

2- Barker DK. Occlusal interferences and temporomandibular dysfunction Gen Dent. 2004;52(1):56-61.

3- Berry DC, Singh BP. Daily variations in occlusal contacts. J Prosthet Dent. 1983;50(3):386-91

4- Bezzon OL, Orsi IA. An interocclusal record made of a combination of wax and acrylic resin. J Prosthet Dent. 1994;72(3):334-6.

5- Calagna LJ, Silverman SI, Garfinkel L. Influence of neuromuscular conditioning on centric relation registrations. J Prosthet Dent 1973;30(4):598-604.

6- Canay S, Cindas A, Uzun G, Hersek N, Kutsal YG. Effect of muscle relaxation splint therapy on the electromyographic activities of masseter and anterior temporalis muscles. Oral Surg Oral Med Oral Pathol Oral Radiol Endod. 1998;85(6):674-9.

7- Capp NJ, Clayton JA. A technique for evaluation of centric relation tooth contacts. Part II: following use of an occlusal splint for treatment of temporomandibular joint dysfunction. J Prosthet Dent. 1985;54(5):697705 .

8- Carlson N, Moline D, Huber L, Jacobson J. Comparison of muscle activity between conventional and neuromuscular splints. J Prosthet Dent. 1993;70(1):39-43.

9- Dworkin SF, LeResche L. Research diagnostic criteria for temporomandibular disorders: Review, criteria, examinations and specifications, critique. J Craniomandib Disord. 1992;6(4):301-55.

10- Ekberg E, Sabet ME, Petersson A, Nilner M. Occlusal appliance therapy in a short-term perspective in patients with temporomandibular disorders correlated to condyle position. Int J Prosthodont. 1998;11(3):2638.

11- Ekberg E, Vallon D, Nilner M. The efficacy of appliance therapy in patients with temporomandibular disorders of mainly myogenous origin. A randomized, controlled, short-term trial. J Orofac Pain. 2003;17(2):1339.

12- Ferrario VF, Sforza C, Tartaglia GM, Dellavia C. Immediate effect of a stabilization splint on masticatory muscle activity in temporomandibular disorder patients. J Oral Rehabil. 2002;29(9):810-5.

13- Fu AS, Mehta NR, Forgione AG, Al-Badawi EA, Zawawi KH Maxillomandibular relationship in TMD patients before and after shortterm flat plane bite plate therapy. Cranio. 2003;21(3):172-9.

14- Gavish A, Winocur E, Ventura YS, Halachmi M, Gazit E. Effect of stabilization splint therapy on pain during chewing in patients suffering from myofascial pain. J Oral Rehabil. 2002;29(12):1181-6.

15- Gilboe DB. Centric relation as the treatment position. J Prosthet Dent $1983 ; 50(5): 685-9$.
16- Glossary of Prosthodontic terms. J Prosthet Dent. 2005;94(1):10-92.

17- Greene CS, Laskin DM. Splint therapy for the myofascial paindysfunction (MPD) syndrome: a comparative study. J Am Dent Assoc. 1972;84(3):624-8

18- Hiyama S, Ono T, Ishiwata Y, Kuroda T. First night effect of an interocclusal appliance on nocturnal masticatory muscle activity. J Oral Rehabil. 2003;30(2):139-45.

19- Kovaleski WC, De Boever J. Influence of occlusal splints on jaw position and musculature in patients with temporomandibular joint dysfunction. J Prosthet Dent. 1975;33(3):321-7.

20- Kuttila M, Bell YL, Savolainen-Niemi E, Kuttila S, Alanen P. Efficiency of occlusal appliance therapy in secondary otalgia and temporomandibular disorders. Acta Odontol Scand. 2002;60(4):248-54.

21- Lucia VO. A technique for recording centric relation. J Prosthet Dent. 1964;14(3):492-505.

22- Okeson JP, Kemper JT, Moody PM. A study of the use of occlusion splints in the treatment of acute and chronic patients with craniomandibular disorders. J Prosthet Dent. 1982;48(6):708-12.

23- Okeson JP, Moody PM, Kemper JT, Halev JV. Evaluation of occlusal splint therapy and relaxation procedures in patients with temporomandibular disorders. J Am Dent Assoc. 1983;107(3):420-4

24- Raphael KG, Marbach JJ, Klausner JJ, Teaford MF, Fischoff DK. Is bruxism severity a predictior of oral splint efficacy in patients with myofascial face pain? J Oral Rehabil. 2003;30(1):17-29.

25- Roark AL, Glaros AG, O’Mahony AM. Effects of interocclusal appliances on EMG activity during parafuncional tooth contact. J Oral Rehabil. 2003;30(6):573-7.

26- Rubinoff MS, Gross A, McCall Junior WD. Conventional and nonoccluding splint therapy compared for patients with myofascial pain dysfunction syndrome. Gen Dent. 1987;35(6):502-6.

27- Singh BP, Berry DC. Occlusal changes following use of soft occlusal splints. J Prosthet Dent. 1985;54(5):711-5.

28- Suvinen TI, Reade PC, Könönen M, Kemppainen P. Vertical jaw separation and masseter muscle electromyographic activity: a comparative study between asymptomatic controls and patients with temporomandibular pain and dysfunction. J Oral Rehabil. 2003;30(8):76572.

29- Torii K, Chiwata I. Relationship between habitual occlusal position and flat bite plane induced occlusal position in volunteers with and without temporomandibular joint sounds. Cranio. 2005;23(1):16-21.

30- Tripodakis AP, Smulow JB, Mehta NR, Clark RE. Clinical study of location and reproducibility of three mandibular positions in relation to body posture and muscle function. J Prosthet Dent. 1995;73(2):190-8.

31- Yap AUJ. Effects of stabilization appliances on nocturnal parafunctional activities in patients with and without signs of temporomandibular disorders. J Oral Rehabil. 1998;25(1):64-8. 